Despite these potential pitfalls, we believe that the EULAR/ ACR criteria are a relevant step forward in appropriately defining who has SLE, and in teaching doctor and medical students how to approach a patient with possible SLE. The data have clearly demonstrated that ANA negative SLE is uncommon, and ANA are a useful entry criterion or screening parameter in case of suspected SLE. The analysis of interaction has important implications in that it has shown interactions within domains, upholding this concept, but not found significant associations between domains (or items in various domains). The latter in fact is an argument that SLE is indeed a disease, not a syndrome, and that it is the effector arm of the autoantibodies in any given SLE patient that underlies the variability.

Conclusions We will need more knowledge on autoantibodies, not less, and probably more clinical training, but this is more of a chance than a challenge. Above all, it has been remarkably easy to work together in this huge group, over the Atlantic and beyond, and I am deeply gratefully for the contribution of so many colleagues. This large team experience of collegiality and friendship will hopefully help the further worldwide collaboration that is necessary for advancing the field.

Acknowledgements The EULAR/ACR SLE classification project was equally supported by EULAR and ACR. Please refer to the publications for contributors to this effort.

\section{THE HISTORY OF LUPUS THROUGHOUT THE AGES}

Laurent Arnaud. Dept. of rheumatology, National French Reference Center for Auto-immune Diseases (RESO), INSERM 1109, Institut PACIFIC, Strasbourg, France

\subsection{6/lupus-2020-eurolupus.6}

The word lupus (Latin term for the wolf) is mentioned for the first time circa $850 \mathrm{AD}$. Originally, the term was used to describe lesions that were said to resemble wolves' bite. Early historical documents have suggested that the term lupus was used indistinctively during the middle-age and the Renaissance for many types of diseases characterized by ulcerous lesions, especially in the lower limbs. In 1230, Rolando of Parma distinguishes 'noli me tangere' (lesions located on the face) from lupula when the lesions are on the limbs. Four centuries later, there is still a great deal of confusion with Sennert, Culpeper \& Cole who mentioned (1661) that '[...] cancer can be divided into 3 species: into the cancer, [...] the noli me tangere $[\ldots]$ and lupus or the wolf if it is in the shins, ankle-bones and thighs'.

The true turning point in the history of lupus occurred in London in the beginning of the 19th century when Robert Willan and his student Bateman published the first atlases of skin diseases (1786-1817), containing the first known representation of a patient with lupus (figure 1). At that time, the disease was classified among 'tubercula' and the distinction between lupus vulgaris (skin tuberculosis) and cutaneous lupus in its modern sense was not clearly made until Keil in 1933. In 1850, the French dermatologist Cazenave mentions for the first time the term 'lupus érythemateux'. Kaposi describes the 'butterfly rash' in 1872 and identifies discoid lupus as a separate entity in 1875 . The history of lupus will take a new turn between 1872 and 1905, with major contributions from Kaposi, Sequiera \& Balean, and Osler who will enable the true recognition of the systemic nature of the disease

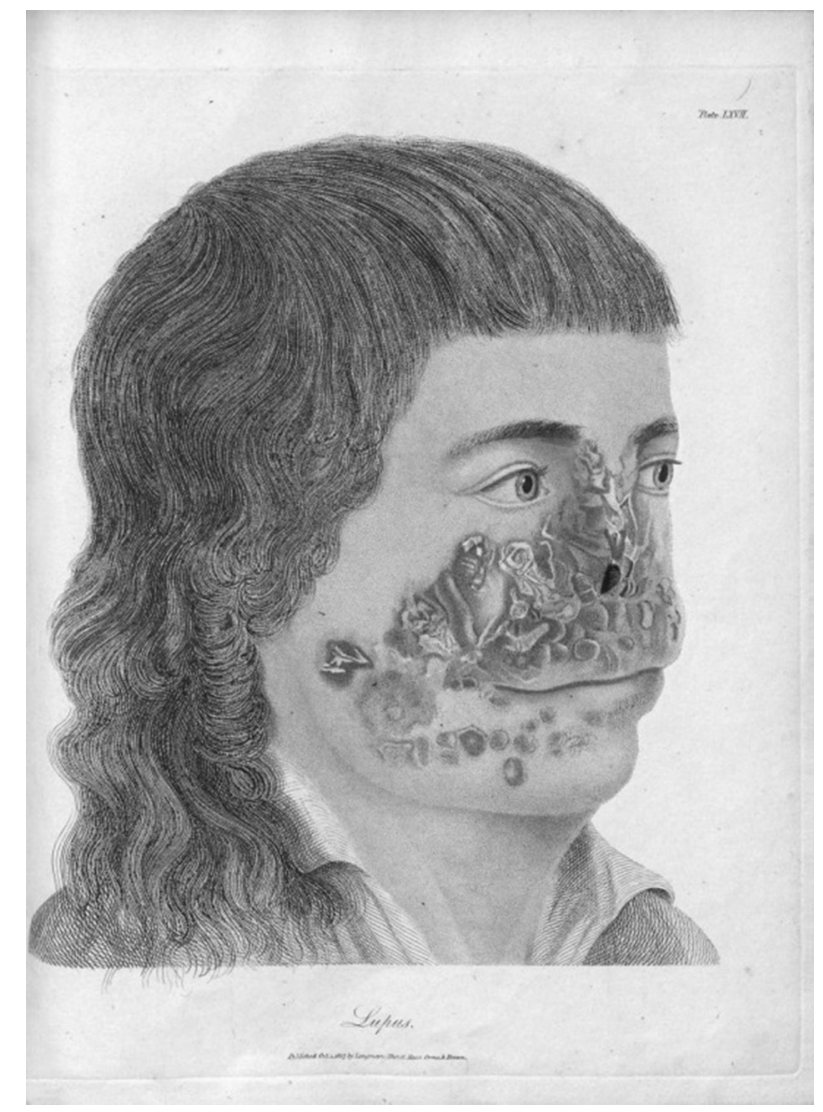

Abstract 16 Figure 1 Delineations of cutaneous diseases. Thomas Bateman, London (1918)

(Systemic Lupus Erythematosus). The modern history of lupus is notably marked by the discovery of lupus cells (LE cells) by Hargraves in 1948, of antinuclear antibodies by Miescher in 1954 and by the recognition of DNA as the main target of ANAs by Seligman in 1957.

Many treatments have been proposed for lupus throughout the ages, including the use of cauterization \& caustics (from the middle age to the modern era), radium (1900-1905), and even concentrated sun light \& UVs in London in 1905! Quinine was introduced in 1894 while most modern treatments for SLE appeared in the second half of the 20th century: glucocorticoids (1948-1952), quinacrine (1951), cyclophosphamide (1954), hydroxychloroquine (1956), azathioprine (1957) and mycophenolate mofetil (1980s). The end of the 20th century and the beginning of the 21st century are marked by a better understanding of the pathogenesis of the disease and the systematic evaluation of treatments, paving the way for improved diagnosis and better care for lupus patients.

\section{PATIENTS EXPECTATIONS, AND WHAT WE (CAN) DO ABOUT IT}

Alain Cornet, Kirsi Myllys, Anne Charlet, Annemarie Sluijmers, Marisa Costa, Elfried Wijsma, Jeanette Andersen. Lupus Europe, UK

\subsection{6/lupus-2020-eurolupus.7}

Background Lupus patient's expectations are no different than everyone else's: 'A better life'. But achieving it requires different steps because it entails (1) A prompt diagnosis, (2) Access to effective treatment with low side-effects, (3) Resolving 
fatigue, depression and the social impact of the disease, and (4) Being involved - individually and collectively.

Methods LUPUS EUROPE is the umbrella organisation that federates, since more than 30 years, national self-help lupus groups around Europe. Through a wide range of initiatives, it seeks to bring alive its vision of 'a better life for people with lupus in Europe, until we reach a world without lupus'.

Results Lupus Europe actively works on all drivers of a better life with lupus. This is constant work in progress with multiparty collaboration

a. Prompt diagnosis - Awareness events are run locally, but more must be done, particularly for GP's, ensuring that lupus signals are identified and testing/referral to expert is done much earlier.

b. Access - Only 54.3\% of European patients are satisfied about their lupus care. Patients expect much awaited new treatments, with a focus on corticoid reduction and targeted therapies for those not responding to standards of care. Lupus Europe engages with academics and industry in more than 20 projects to better understand the disease and design better treatments; and with ERN-ReCONNET to bring best available care to all in Europe. The Patient Advisory Network is available to support your projects with significant patient's added value.

c. Fatigue, depression and social impact: treating lupus is one thing, but even when lupus is controlled, a significant proportion of patients continue to claim debilitating fatigue, depression, or the inability to participate fully in a social life. Much remains to be understood and resolved. Lupus Europe will conduct a survey 'living with lupus in 2020' to measure evolution since our 2010 survey. In the meantime, we are not powerless: (i) Lupus Europe designed an exercise program, endorsed by the ERN to help patients start fight fatigue with exercise, regardless of their current fitness level, and (ii) practical tips based on social psychology and patient experience can help fight depression.

d. Being involved - Individually: Our patient panel on adherence highlighted that being involved in the decision making (feeling listened to, understanding the disease/ treatment, and shifting from 'YOUR prescriptions to OUR treatment plan') is key to increase adherence.

e. Being involved - Collectively: Patients are increasingly ready to be involved in the fight against lupus and to be trained to help. 'Nothing about us without us' is not a political motto, but a strong desire to be part of the solution. Our experience shows that involving patients in research is a triple win: win for the project, (funding or process often requires patient involvement), win for the team (new insights and out of the box thinking from collaborating patients), and win for the patient (being involved has a therapeutic effect and hopefully results in a better end product/treatment). Many patients do not know how they can be involved in research close to their homes. When options are explained by a person they trust (\#1 is their rheumatologist), a majority is interested in taking part. A key area of work to concentrate on is to train both patients and researchers so that patient involvement is not perceived as an additional burden in an already very full schedule, but a valuable help, even very early in the process.

Interestingly, interactions with patients repeatedly highlight the precious link established between patients and their lupus specialist. Patients are incredibly thankful to their doctors for their commitment, knowledge and relentless efforts to support them, so often way beyond professional obligations. One can say that one of the patient expectations is also to have a 'happy rheumatologist', and there also, we are willing to help. Conclusions Unsurprisingly, Patient's expectations are to have 'A better life'. Working together, much progress has already been made, and LUPUS EUROPE is committed to keep working on this, together with all partners.

Acknowledgements Lupus Europe is mostly financed by Contributions of Industry - GSK, UCB, Idorsia, Boehringer, Janssen, Leo Pharma

\section{I8 THE ROLE OF THE INTERFERON SYSTEM IN SLE}

Lars Rönnblom. Dept. of Medical Sciences, Uppsala University, Sweden

\subsection{6/lupus-2020-eurolupus.8}

The interferon (IFN) system can be defined as the IFN genes and proteins, the inducers of IFN production, the IFN producing cells, as well as target cells affected by IFN. There are three different types of IFN (I-III), and type I IFN is the largest family consisting of more than 15 different proteins. The type I IFNs are our main defense against viral infections and production can be triggered by ligation of several different sensors of nucleic acid. Most cells can produce small amounts of type I IFN, but the principal type I IFN-producing cell is the plasmacytoid dendritic cell (pDC).

There are several observations suggesting an important role for the IFN system in the etiopathogenesis of SLE, but also other autoimmune diseases. Among these are the reported development of SLE during treatment with IFN- $\alpha$, a prominent increase in the expression of IFN regulated genes (an IFN signature) in SLE, the existence of endogenous, or self derived, IFN inducers in SLE patients and a genetic association between SLE and gene variants within the type I IFN signaling pathway.

Several studies have shown that type I IFN seems to be the most important IFN for inducing the IFN signature, but it's clear that type II and type III IFNs also can contribute. Furthermore, patients with SLE have epigenetic changes in type I IFN regulated genes, which are hypomethylated. Thus, there are strong evidences that activation of the IFN system, and particular the type I IFNs, is a key event in the SLE disease process.

The type I IFN system is closely connected to a number of other cytokine and chemokine pathways, which all can contribute to both the IFN signature and type I IFN effects. Important type I IFN effects are maturation and differentiation of dendritic cells, activation of $\mathrm{T}$ and $\mathrm{B}$ cells with enhanced antibody production and induction of increased expression of autoantigens. Consequently, type I IFNs can act as an immune adjuvant and promote an autoimmune process. Furthermore, the regulation of the type I IFN system is abnormal in SLE and negative feedback mechanisms are impaired, causing a positive feed-forward loop, which sustain the autoimmune reaction. Many different therapeutic targets exist within the IFN system and several studies have recently been published showing beneficial effects of blocking or down-regulating the activated type I IFN system in SLE. However, not all patients with SLE improve during IFN inhibition and obviously; better stratification of patients and more precise treatments are needed in the heterogeneous group of SLE 